\title{
Static and dynamic experimental investigations of a micro-electromechanical cantilever in air and vacuum
}

\author{
S. Gutschmidt ${ }^{1}$, V. Rochus ${ }^{2}$, J.-C. Golinval ${ }^{2}$ \\ ${ }^{1}$ University of Canterbury, Department of Mechanical Engineering, Christchurch, New Zealand \\ phone: +64-(0)3-364 2987 ext.7215, e-mail: stefanie.gutschmidt@ canterbury.ac.nz \\ ${ }^{2}$ University of Liège, Department of Aerospace and Materials, Liège, Belgium \\ phone: +32-(0)4-366 4853, e-mail: v.rochus@ulg.ac.be, jc.golinval@ulg.ac.be
}

\begin{abstract}
The dynamic response of electro-mechanically actuated micro structures is governed by nonlinear effects which directly influence their performance. To date, most work in the field of micro/nano systems is done experimentally and documented theoretical research consists of nonlinear lumped-mass models as well as continuum mechanics approaches. Existing and codified computational tools for micro-electromechanical systems (MEMS) more and more penetrate the market of designers and fabrication of such devices. The present work is the experimental part of a bench-mark study of a selected microbeam structure investigated with aforementioned analytical and computational techniques and compared to experiments performed with a Polytec MSA400 analyzer. The emphasis of this benchmark study is put on the nonlinear dynamic behavior with respect to qualitative and quantitative explanations of the electro-mechanical actuation and damping mechanisms.
\end{abstract}

\section{Introduction}

The broad field of research on micro- and nanoelectromechanical systems (MEMS/NEMS) is addressed by various approaches. Foremost among the methods used and developed for MEMS/NEMS is the finite-element method/analysis (FEM/FEA) [2, 17]. Applications of MEMS can be accelerated by implementation of computer aided design (CAD) tools, optimization and parametric studies for which, at least in the static case, finite-element models are ideally suited since they are able to capture the complexity of such intentions. Prior to the FEA and also to reduced-order model analysis in general, is an approximation technique based on an initial boundary value problem formulation which codifies experimental observations. The design of MEMS devices demands a careful and detailed modeling, while special attention must be given to the coupled fields present in such devices (electric, magnetic, elastic, thermal, etc.), like the damping mechanisms and accompanying boundary conditions of the structure. Lumped-mass approaches have shown to be insufficient to describe the observed behavior quantitatively [6]. Thus, this work focuses on experimental observations of a microcantilever (see Fig. 1) that show the evidence of the nonlinear behavior. The present work serves as systematic guideline for deriving a mathematical model which is able to codify the nonlinear behavior and allows for quantita-

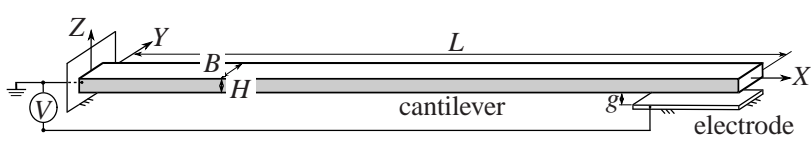

Figure 1: Definition sketch of the micro-cantilever system.

tive and qualitative predictions of the system behavior. At this point, we would like to refer to another work of our group which will be published in short. This work will be the second part of the aforementioned bench-mark study and evaluates proposed theoretical approaches with respect to experimental observations shown throughout this work. The principal aim of the bench-mark study is to be able to contribute to some of the remaining questions related to MEMS modeling, including the full understanding of (squeeze-film) damping and actuation mechanisms in such systems.

\section{Experimental setup}

Experimental setups are designed by the third-party contractor Institut d'Electronique Fondamentale (IEF), a research centre in Paris, France, and fabricated by the subcontractor of IEF, MEMSCAP, within the framework of the project "Action de Recherche Concertée". The PolyMUMPS technology has been used for fabricating the MEMS devices. The design testing and earlier static experiments have been performed by IEF, of which selective results are presented in this work. The fabrication process itself can be found in literature [16, 3]. The present work considers a micro cantilever as shown in Fig. 1. The design and fabrication of this micro structure has been done within the framework of the aforementioned project "Action de Recherche Concertée". Details on fabrication for

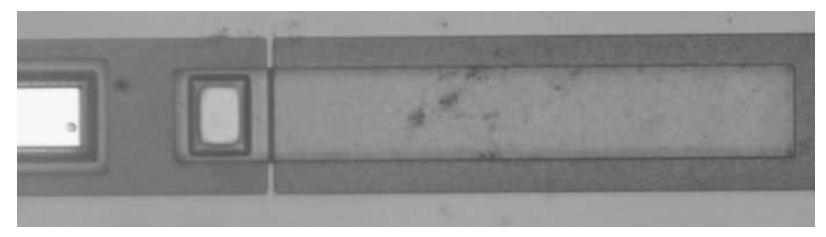

Figure 2: Magnified view of the micro cantilever $(175 \mu \mathrm{m} \times 30 \mu \mathrm{m} \times 1.9 \mu \mathrm{m})$. 
this micro cantilever as well as design specifications and tolerances are documented in [11]. The cantilever's dimensions are $175 \mu \mathrm{m} \times 30 \mu \mathrm{m} \times 1.9 \mu \mathrm{m}(L \times B \times H)$. Fig. 2 shows the micro structure while being placed under the microscope. Note, that the bottom electrode, unlike shown in the sketch in Fig. 1 is implemented in full length with the cantilever structure.

The micro structure cantilever is chosen, because of its structural simplicity, and the absence of mid-planestretching and prestress physics, which significantly matters when it comes to identifying parameters and tracking after the origin of mismatching characteristics in the evaluation analysis later on.

\subsection{Identification of parameters}

The material properties and dimensions of the micro cantilever are taken from the specification of the fabrication [11]. However, the true dimensions of the structure are obtained from a performed profilometry. Fig. 3 shows, representatively, the measurements of a cantilever of length $100 \mu \mathrm{m}$ (specification value) which is of the same production line as the considered cantilever of length $175 \mu \mathrm{m}$. According to the profilometry of this cantilever the real gap
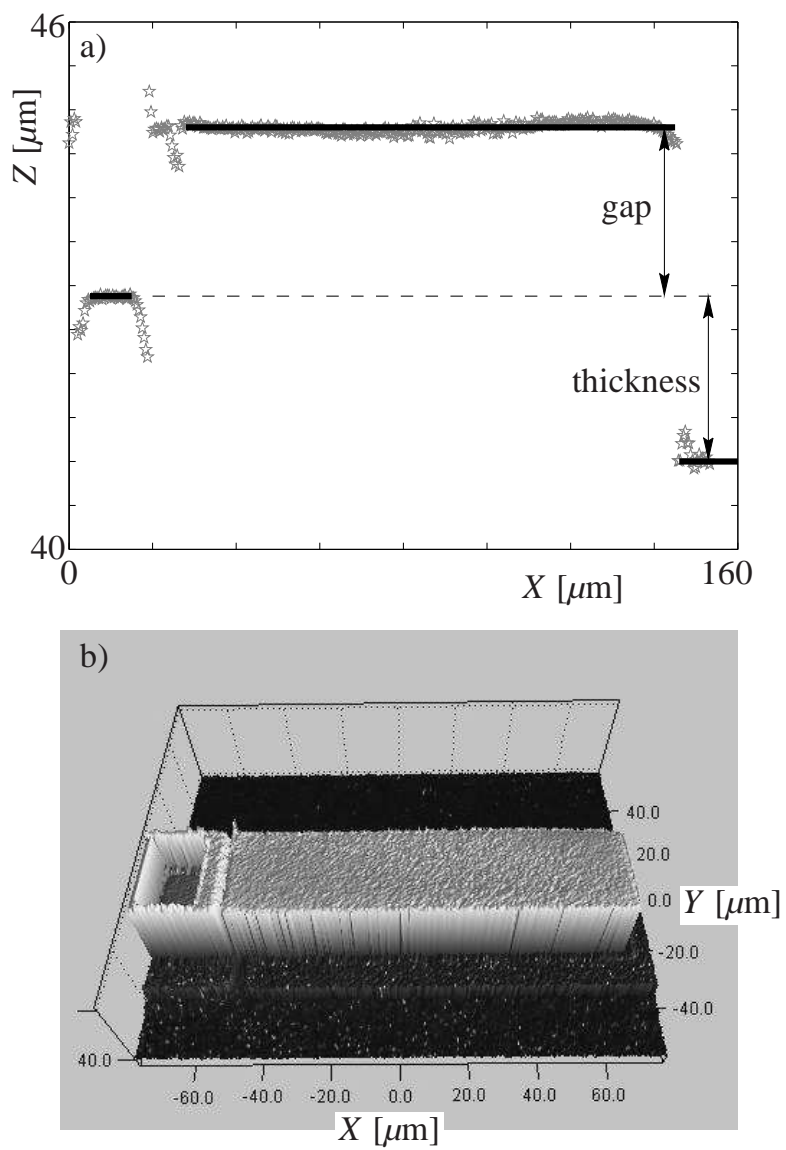

Figure 3: Profilometry of the cantilever with the specification dimensions $100 \mu \mathrm{m} \times 30 \mu \mathrm{m} \times 1.9 \mu \mathrm{m}$; a) $Z-X$ plane b) $3 \mathrm{D}$ view; stars in a) denote measured points, lines mark distinct Z-levels. and thickness are identified as $g=2 \mu \mathrm{m}$ and $H=1.9 \mu \mathrm{m}$. Profilometry of six additional cantilevers (of the same fabrication charge) confirm such values (by two digits after the comma). The specification tolerances for the gap and thickness are $\pm 0.25 \mu \mathrm{m}$ and $\pm 0.15 \mu \mathrm{m}$, respectively [11]. The length and width of the structure fall into similar tolerances as the thickness and gap. However, they are less critical with respect to the influence of the static and dynamic behavior.

\subsection{Experimental observations}

\subsubsection{Static investigations}

The experimental static analysis consists of quasistatic measurements of the deflection while the input voltage is increased. The POLYTEC MSA400 analyzer in our laboratory is equipped with the velocity encoder. Thus, the static measurements are replaced by corresponding quasistatic experiments, i.e. the DC voltage is set to zero and the AC voltage is applied at a much lower frequency (e.g. 2 $\mathrm{kHz}$ ) than the first natural frequency of the system. In the linear case (for small deflections) the amplitude response at twice the excitation frequency and for the root-meansquare value of the input voltage is then equivalent to the static measurement. The MSA400 analyzer in our laboratory allows for a maximum total input voltage of $10 \mathrm{~V}$, i.e. quasistatic measurements up to $10 / \sqrt{2} \mathrm{~V}=7.07 \mathrm{~V}$ are performed. Fig. 4 depicts the measured maximum deflec-

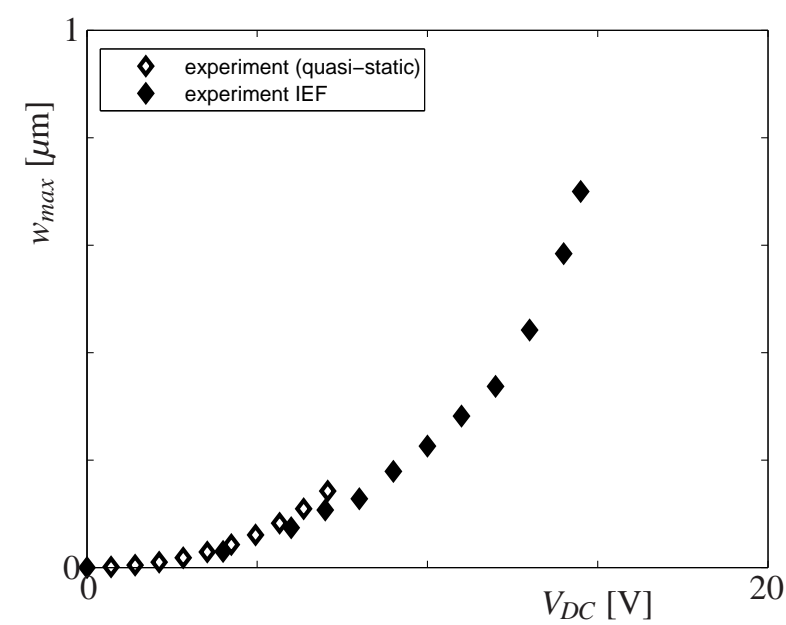

Figure 4: Measured static equilibrium characteristics of the cantilever.

tions of the cantilever with increasing DC-voltages. Solid diamonds represent static measurements performed by IEF [11]. The quasi-static measurements performed in our laboratory (empty diamonds), however, must be handled with care. While for the linear range, both characteristics stand in good agreement, for higher DC voltages (resulting in larger beam deflections) the curves diverge distinctively. This difference matters increasingly towards the structure's instability, known as pull-in point [18]. 


\subsubsection{Dynamic investigations}

Dynamical investigations of the electromechanically coupled cantilever system include several frequency-response and phase plots. Fig. 5 portraits three frequency responses (and corresponding phase plots) of one and the same cantilever at room temperature and under atmospheric pressure. The excitation is a white noise signal having applied
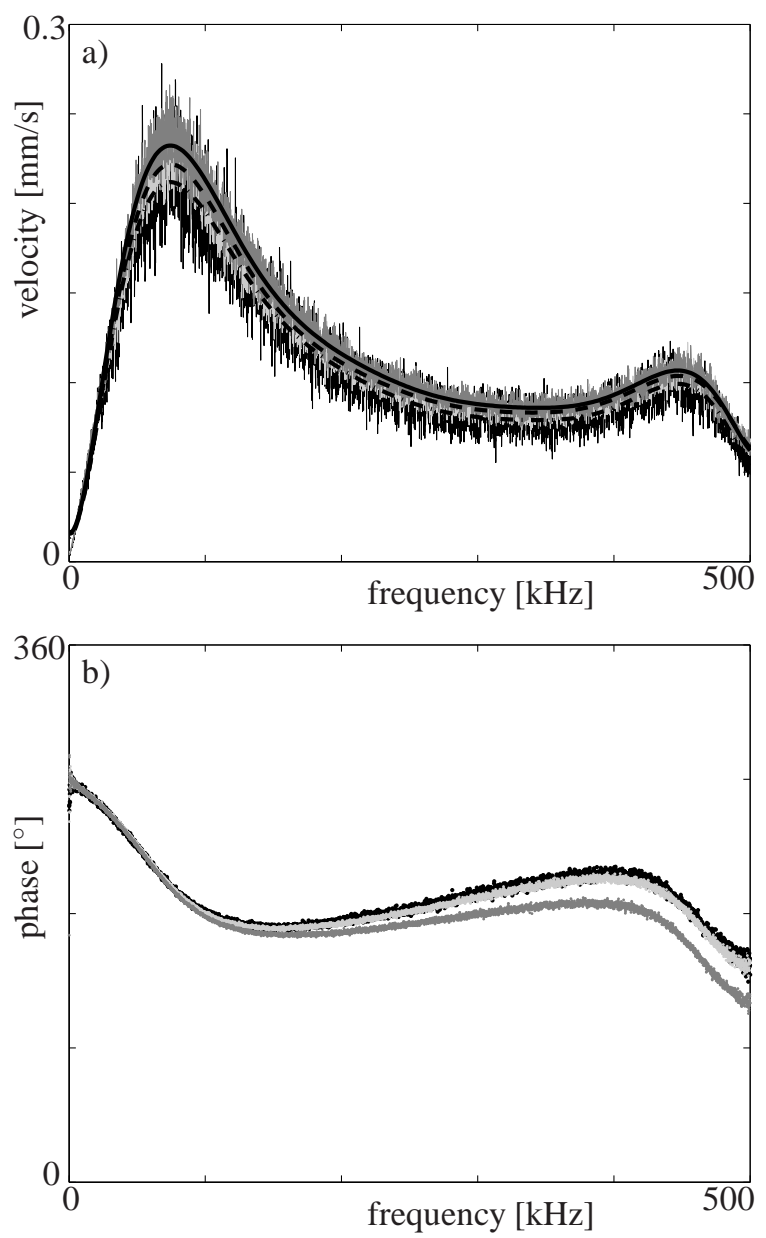

Figure 5: Three frequency responses of one cantilever under similar surrounding and actuation conditions, i.e. atmospheric pressure, room temperature, input signal: white noise (offset voltage $5 \mathrm{~V}$, random p-p input voltage $5 \mathrm{~V}$ ), a) magnitude response of the velocity $[\mathrm{mm} / \mathrm{s}]$, b) phase shift $\left[{ }^{\circ}\right]$.

an offset and a random signal of $5 \mathrm{~V}$, respectively. Fig. 6 shows the evenly distributed signal in the frequency domain over a frequency band of $500 \mathrm{kHz}$. Note, that the average value of the distributed input voltage per Hertz is approximately $28 \mathrm{mV}$. The purpose of the three measurements, of one and the same experiment in Fig. 5, is to show the magnitude of divergency within repeating performances. According to the deviation plot in Fig. 7, the amplitudes vary the most in and near resonances. Furthermore, we observe that repetitions of such measurements in air could definitely show a deviation of amplitudes of $\approx 4 \%$, while a

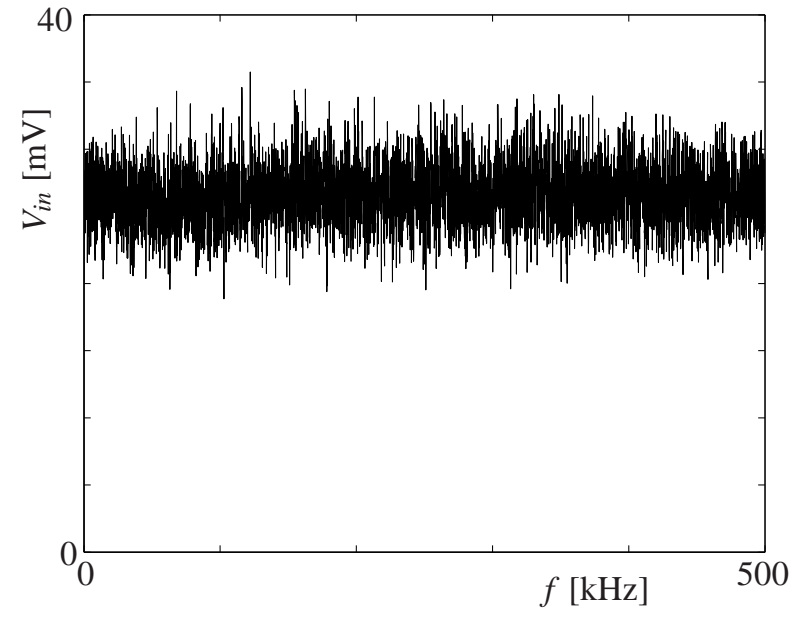

Figure 6: Input signal (white noise) depicted in the frequency domain, offset voltage $5 \mathrm{~V}$, noise p-p signal $5 \mathrm{~V}$, frequency band $500 \mathrm{kHz}$.

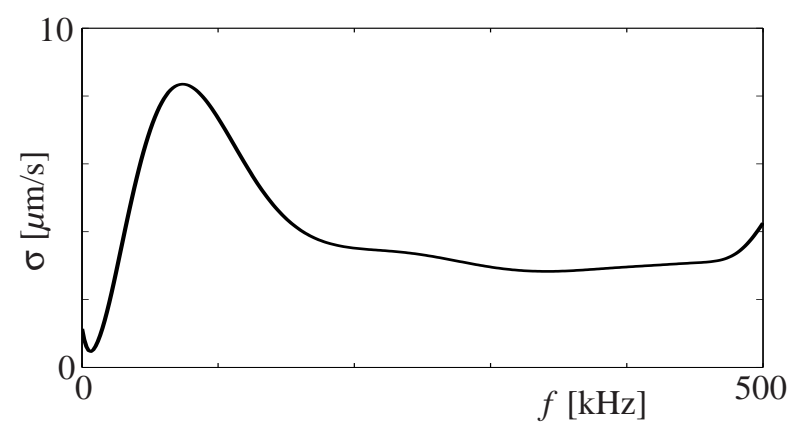

Figure 7: Standard deviation obtained from the three meanvalue splines in Fig. 5a

shifting of natural frequencies is not noticed. However, experimental results for higher frequencies are handled with care according to the bad coherence function in this region (see Fig. 8).

In the following we show the dynamical behavior of the same cantilever in vacuum for several air pressures and noise input voltages. The nonlinear behavior of such MEMS, as frequently reported in literature [20, 10], is also observed in the considered cantilever of this work. Fig. 9 portraits the frequency responses, and accordingly the phase plots, for three different noise p-p input voltages, namely $1 \mathrm{~V}, 3 \mathrm{~V}, 5 \mathrm{~V}$. (Notice the commonly used scaling of the vertical axis in order to track nonlinear phenomena.) The hardening behavior of the cantilever becomes more evident with increasing input voltages. Furthermore, also the jump phenomena is recognized. Unfortunately, a sweep-up/down characteristic of this cantilever cannot be presented alongside. (During one of the dynamic measurements the cantilever pulled into the bottom electrode and broke.)

The dynamical behavior of the cantilever under various pressure levels is considered next. Fig. 10 portraits the frequency responses (and phase plots) for three different 


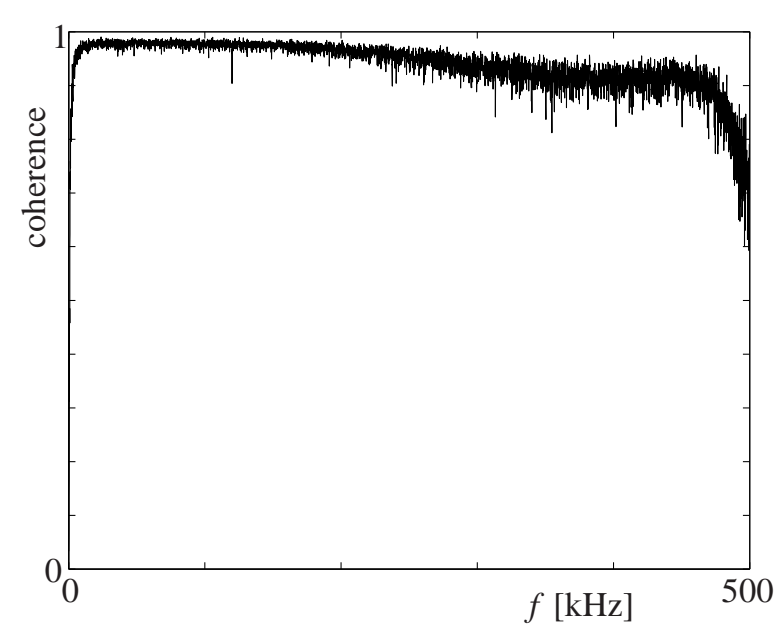

Figure 8: Coherence of one of the measurements (black line) shown in Fig. 5

pressures as well as the response in air. According to the responses shown in Fig. 10a the first and second eigenfrequency is identified to be at $f_{1}=73.1 \mathrm{kHz}$ and $f_{2}=465.8$ $\mathrm{kHz}$, respectively. We observe additional peaks at lower amplitudes (see zoom-in plot Fig. 10b), which appear to be integers of the peak of the first frequency. A simple linear one-degree-of-freedom approach, while having matched the first natural frequency by tuning the Young's modulus to $E=123.8 \mathrm{MPa}$, suggests the second frequency to be at $f_{2}=457.8 \mathrm{kHz}$ (1DOF). Compared to the experimental value of $f_{2}=465.8 \mathrm{kHz}($ exp.) the linear estimate mismatches the experimental value by less than two percent, which needs to be evaluated. Note, that, also in vacuum, repeated measurements may show deviations in amplitudes while deviations of frequencies are not recognized (c.f. Fig. 10). The responses at the the first natural frequency (Fig. 10a) shows a fairly sharp peak which could lead to the wrong conclusion, that the behavior appears to be linear. Fig. 11 depicts a zoom-in of the response curve in (Fig. 10a) at the first natural frequency. The nonlinear behavior, depicted by the tendency of the hardening behavior in Fig. 11, is confirmed by looking at the coherence function at this frequency range, Fig. 12. While in the linear and an ideal averaging case of sampling numbers going to infinity the coherence function is one, the poor coherence function shown here is but another indicator of the existence of a nonlinear behavior of the micro-cantilever system. (All considered experiments throughout this manuscript have been sampled with $N=50$.)

\section{Concluding remarks}

The presented work shows nonlinear phenomena in all measurements, static as well as dynamic. Uncertainties, which relate to repetitions of experiments, are within four percent for amplitude deviations. No deviations of frequency shifting are noticed, neither in air nor in vacuum. Based on the experimental observations, presented in this
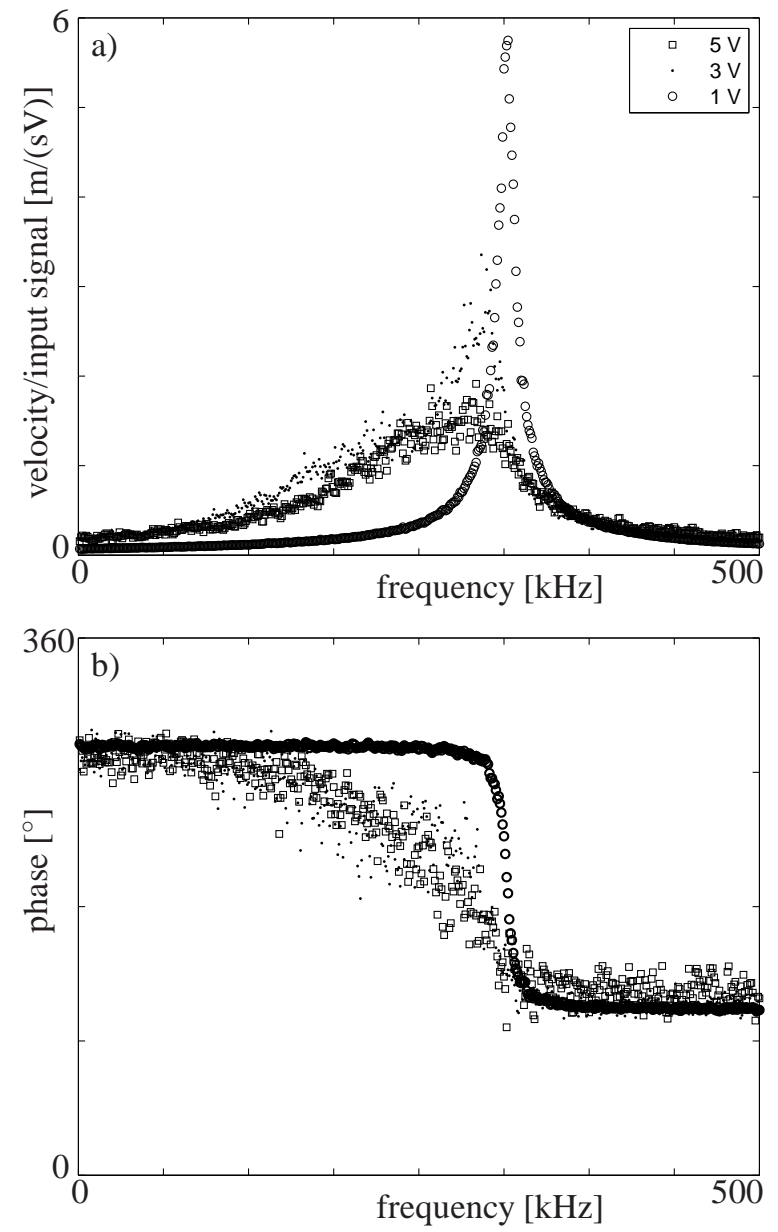

Figure 9: Frequency responses of the micro-cantilever at a pressure of $2.4 \cdot 10^{-4}$ mbar and room temperature, input signal: white noise (offset voltage $5 \mathrm{~V}$ ), a) magnitude responses of the velocity $[\mathrm{mm} / \mathrm{s}]$, b) phase shifts $\left[{ }^{\circ}\right]$.

work, we propose to derive a consistent nonlinear continuum model in support with a computational finite-element model (using the in-house made OofELIE solver), which is able to predict the dynamic behavior of the considered cantilever system in quality and quantity within the bounds of remaining fabrication uncertainties. The resulting systematic bench-mark study is proposed to serve as a guideline for design and the modeling of MEMS in general (but foremost for micro-structures which are having a similar geometric complexity).

\section{Acknowledgments}

We wish to express special thankfulness to two supporting companies. First, our thanks goes to K. Fleury from Centre Spatial de Liège, who did the profilometry measurements and second, we which to acknowledge the ongoing support from Microsys laboratories (Liège). Fig. 2 is taken with one of their equipments.

This work is part of the larger project Modélisation, Simulation multiphysique et optimisation de 
problèmes couplés: Application aux micro-systèmes électromécaniques. The first author wishes to express her gratitude to the Belgian Federal ScIENCE Policy OFFICE for the financial support during her postdoctoral appointment. The second author acknowledges the financial support of the BELGIAN NATIONAL FUND FOR SCIENTIFIC RESEARCH.

\section{References}

[1] Krylov, S., Int. J. of Nonlin. Mech., 42, 626 (2007)

[2] Rochus, V., Rixen, D. J., Golinval, J.-C., Int. J. Numer. Meth. Engng, 65, 461 (2006)

[3] Rochus, V., PhD thesis, ULg Liège, Belgium (2006)

[4] Younis, M.I., Abdel-Rahman, E.M., Nayfeh, A., J. Microelectromech. Syst., 12 (5), 672 (2003)

[5] Buks, E., Roukes, M.L., J. Microelectromech. Syst., 11 (6), 802 (2002)

[6] Lifshitz, R., Cross, M.C., Phys. Rev. B, 67 (134302), $1-12(2003)$

[7] Despont, M., Drechsler, U., Yu, R., Pogge, H.B., Vettiger, P., J. Microelectromech. Syst., 13 (6), 895 (2004)

[8] Zavracky, P.M., Majumder, S., McGruer, N.E., J. Microelectromech. Syst., 6, 3 (1997)

[9] Senturia, S.D., Harris, R.M., Johnson, B.P., Kim, S., Nabors, K., Shulman, M.A., White, J.K., J. Microelectromech. Syst., 1, 3 (1992)

[10] Tilmans, H.A., Legtenberg, R., Sens. Actuators, A45, 67 (1994)

[11] Parrain, F., 'Rapport d'avancement pour l'année académique 2004-2005", IEF-CNRS UMR 8622 (2005)

[12] Hung, E.S., Senturia, S.D., J. Microelectromech. Syst., 8 (3), 280 (1999)

[13] Hornstein, S., Gottlieb, O., Nonlinear Dyn., DOI 10.1007/s11071-008-9335-5

[14] Gutschmidt, S., Gottlieb, O., In Proc. of 6th EUROMECH Nonlinear Dynamics Conference, St. Petersburg, Russia, June 30-July 4, 2008

[15] Griffiths, D.J., Introduction to Electrodynamics, Englewood cliffs, NJ: Prentice-Hall (1981)

[16] Madou, M.J., Fundamentals of Microfabrication, 2nd edition, CRC Press LLC (2002)

[17] Gabbay, L.D., Mehner, J.E., Senturia, S.D., J. Microelectromech. Syst., 9, 262 (2000)

[18] Nayfeh, A.H., Younis, M.I., Abdel-Rahman, E.M., Nonlinear Dyn., 41, 211 (2005)
[19] Krylov, S., Maimon, R., J. Vib. Acoust., 126, 332 (2004)

[20] Senturia, S.D., Microsystem Design, Kluwer Academic Publishers, Bosten (2001)

[21] Nayfeh, A.H., Nonlinear Interactions, WileyInterscience, New York (2000)

[22] Shabana, A.A., Theory of Vibration, Springer-Verlag, New York (1991)

[23] Gottlieb, O. and Champneys, A.R., IUTAM Chaotic Dynamics and Control of Systems and Processes in Mechanics, Springer, 117 (2005)

[24] Osterberg, P.M., Senturia, S.D., IEEE J. MEMS, 6, 107 (1997)

[25] McCarthy, B., Adams, G.G., McGruer, N.E., Potter, D. IEEE J. MEMS, 11, 276 (2002) 

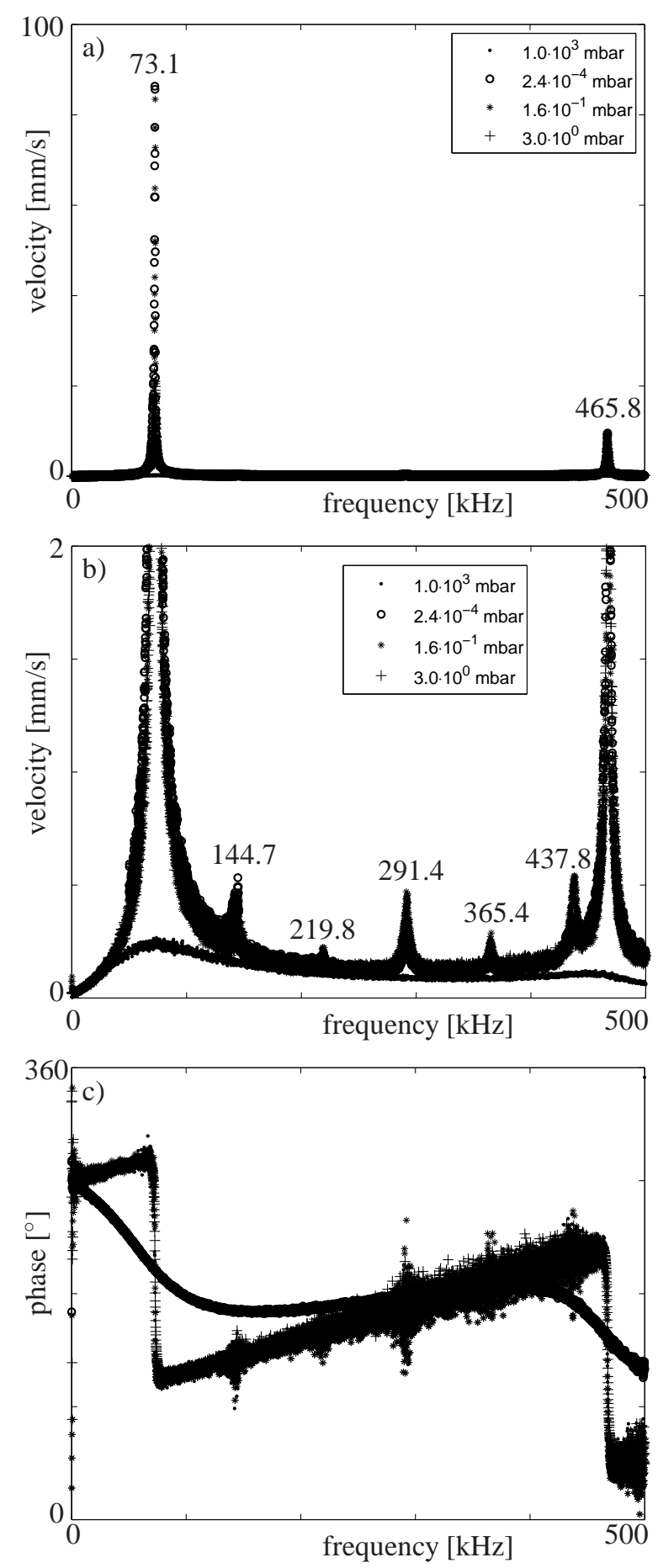

Figure 10: Frequency responses of the micro-cantilever at different pressure levels and room temperature, input signal: white noise (offset voltage $5 \mathrm{~V}$, noise p-p $5 \mathrm{~V}$ ), a) magnitude responses of the velocity $[\mathrm{mm} / \mathrm{s}], \mathrm{b})$ zoom-in of a), c) phase shifts $\left[^{\circ}\right]$.
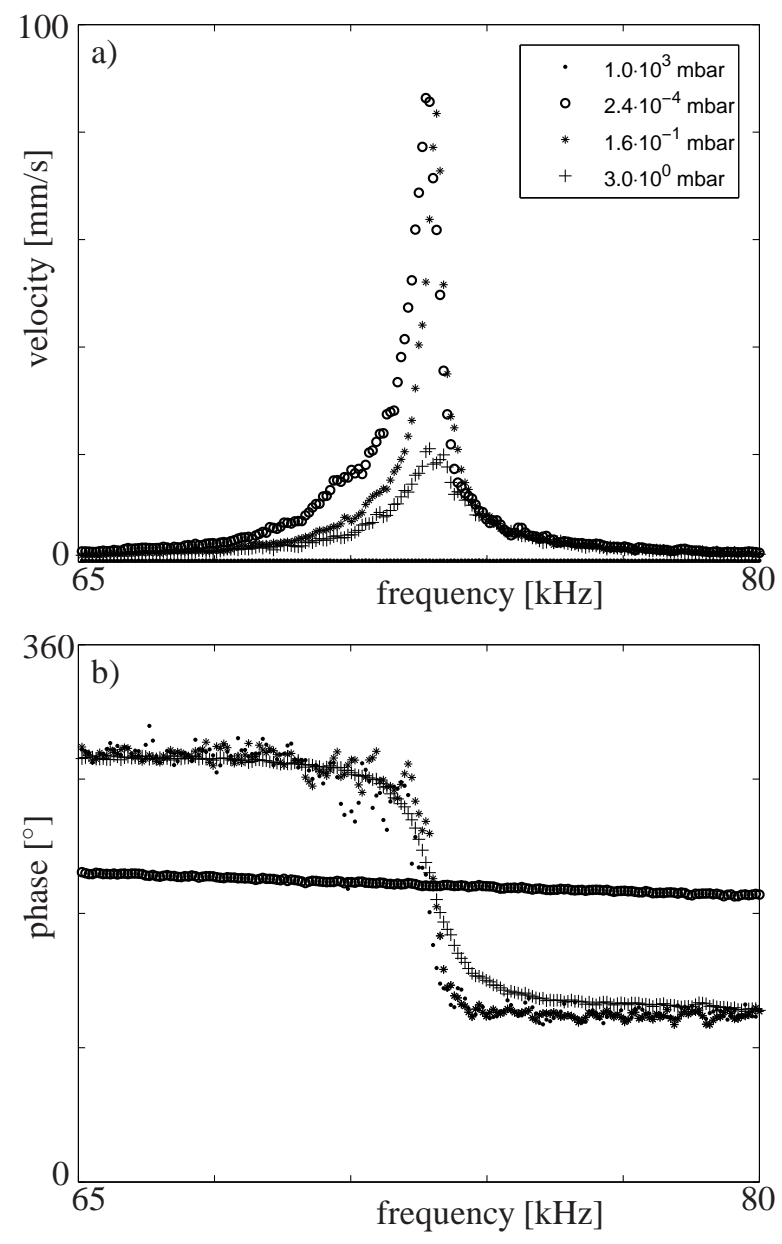

Figure 11: Zoom-in plot of Fig. 10a.

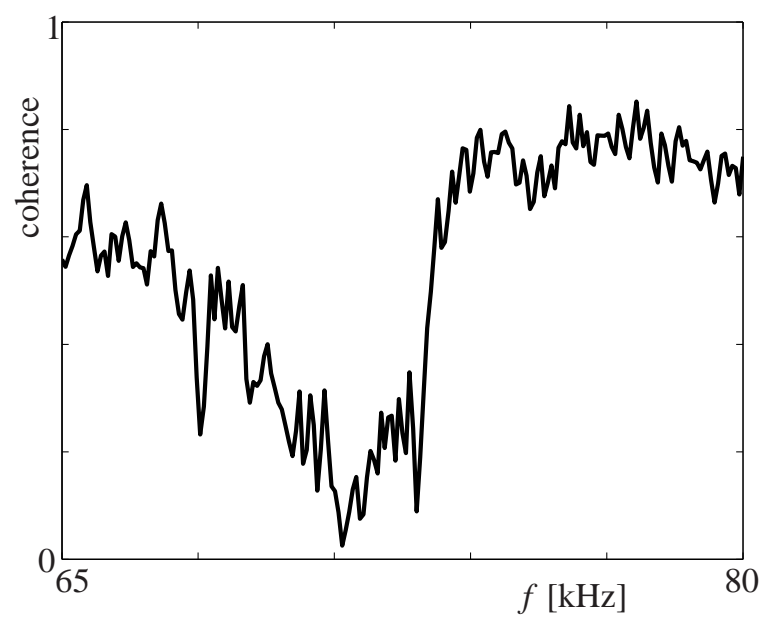

Figure 12: Coherence of the response in Fig. 11 for the air pressure of $2.4 \cdot 10^{-4} \mathrm{mbar}$ 